\title{
Chemokine (C-X-C) Ligand 12 Facilitates Trafficking of Donor Spermatogonial Stem Cells
}

\author{
Zhiyv Niu, ${ }^{1,2}$ Shaun M. Goodyear, ${ }^{1}$ Mary R. Avarbock, ${ }^{1}$ and Ralph L. Brinster ${ }^{1}$ \\ ${ }^{1}$ Department of Animal Biology, School of Veterinary Medicine, University of Pennsylvania, Philadelphia, PA, USA \\ ${ }^{2}$ Department of Medical Genetics, Mayo Clinic, Rochester, MN, USA \\ Correspondence should be addressed to Ralph L. Brinster; brinster@vet.upenn.edu
}

Received 14 October 2015; Accepted 29 December 2015

Academic Editor: Bernard A. J. Roelen

Copyright (c) 2016 Zhiyv Niu et al. This is an open access article distributed under the Creative Commons Attribution License, which permits unrestricted use, distribution, and reproduction in any medium, provided the original work is properly cited.

\begin{abstract}
The chemokine (C-X-C) receptor type 4 (CXCR4) is an early marker of primordial germ cells (PGCs) essential for their migration and colonization of the gonads. In spermatogonial stem cells (SSCs), the expression of CXCR4 is promoted by the self-renewal factor, glial cell line-derived neurotrophic factor (GDNF). Here, we demonstrate an important role of CXCR4 during donor mouse SSCs reoccupation of the endogenous niche in recipient testis. Silencing of CXCR4 expression in mouse SSCs dramatically reduced the number of donor stem cell-derived colonies, whereas colony morphology and spermatogenesis were comparable to controls. Inhibition of CXCR4 signaling using a small molecule inhibitor (AMD3100) during the critical window of homing also significantly lowered the efficiency of donor-derived SSCs to establish spermatogenic colonies in recipient mice; however, the self-renewal of SSCs was not affected by exposure to AMD3100. Rather, in vitro migration assays demonstrate the influence of CXCR4-CXCL12 signaling in promoting germ cell migration. Together, these studies suggest that CXCR4-CXCL12 signaling functions to promote homing of SSCs towards the stem cell niche and plays a critical role in reestablishing spermatogenesis.
\end{abstract}

\section{Introduction}

The spermatogonial stem cell (SSC) is the foundation of an elegant system for the transmission of genetic material, as functional differentiation produces an exponential number of sperm carrying the haploid genome $[1,2]$. Identifying the intrinsic and extrinsic factors regulating SSCs and their niche is important to understanding mechanisms regulating SSC self-renewal and differentiation and offers significant translational benefits towards treating male infertility, especially in regard to pediatric oncology where there is growing concern over the gonadotoxic side effects of radiation and chemotherapy on the fertility of prepubertal boys [1-3].

While considerable effort has been made to understand the SSC niche in the testis, there is still much that remains unknown. The spatial localization of SSCs within the testis niche is in part supported by Sertoli, peritubular myoid, and Leydig cells, as well as microvasculature, which all work in concert to produce soluble factors and extracellular matrix components that provide cues for self-renewal and differentiation $[2,4]$. One example is Sertoli cell and peritubular myoid cell production of the soluble ligand, glial cell line-derived neurotropic factor (GDNF), which is a crucial factor regulating SSC self-renewal [5-8]. GDNF elicits its effects via the GDNF family receptor $\alpha 1$ (GFR $\alpha 1)$ and regulates several downstream factors, including ets variant 5, B-cell CLL/lymphoma 6 member B, LIM homeobox 1, and microRNA-21; all of which are important in mediating SSC homeostasis $[9,10]$. In addition to GDNF, several other factors including the chemokine (C-X-C motif) ligand 12 (CXCL12, also referred to as SDF-1) are shown to aid in maintaining the SSC niche [11-13].

CXCL12 is the cognate ligand for the chemokine (C-XC) receptor type 4 (CXCR4), a G protein-coupled receptor (GPCR) [14]. Studies show that the CXCR4-CXCL12 signaling axis is important in promoting chemotaxis in a variety of cell types, including the recruitment and retention of bone marrow and germline stem cells to their respective niches $[15,16]$. Notably, CXCL12 concentration gradients produced by the mesenchyme of the genital ridge recruit 
CXCR4 expressing primordial germ cells to colonize the gonads [16]. The importance of CXCR4-CXCL12 signaling in maintaining SSCs was unclear until recent studies showed that perturbation of CXCR4-CXCL12 signaling in the testis of recipient mice using a small molecule inhibitor, AMD3100, drastically reduced donor-germ cell colonization [12]. Further evaluation of this pathway has revealed that silencing CXCR4 expression in SSCs, or depletion of the CXCR4 expressing SSC population, greatly reduces the colonizing potential of donor-derived germ cells in recipient testes [11, 12]. In this study we elaborate on the contribution of CXCR4CXCL12 signaling and further examine its ability to promote effective homing of SSCs to their stem niche.

\section{Methods}

2.1. Germ Cell Isolation and Culture. Mouse SSC-enriched germ cell cultures were established from C57BL/6 (Stock no. 000664; The Jackson Laboratory) or ROSA26 (Stock no. 002073; The Jackson Laboratory) mice as previously described [17]. In brief, the testes from postnatal day 68 pups were collected and digested with $0.25 \%$ trypsinethylenediaminetetra-acetic acid (EDTA; Life Technologies) containing $7 \mathrm{mg} / \mathrm{mL}$ of DNase I (Sigma), followed by removal of nonviable cells and debris by centrifugation on a $30 \%$ Percoll gradient $\left(600 \times \mathrm{g}, 4^{\circ} \mathrm{C}, 7 \mathrm{~min}\right)$. A microbead-conjugated antibody against THY1.2 (Miltenyi Biotec) was used to enrich the SSC fraction and these cells were cultured on mitotically inactivated SIM mouse embryo-derived thioguanine- and ouabain-resistant (STO) feeders at a density of $0.5-1.0 \times 10^{5}$ cells/well. The SSC-enriched germ cell cultures were maintained in a previously described chemically defined, serumfree minimal essential medium alpha $(\mathrm{MEM} \alpha)$ medium (mSFM) [17]. The medium was replaced every 2-3 days and germ cell cultures were passaged approximately every 7 days. For in vitro quantification of SSCs, germ cell cultures were digested using trypsin-EDTA and the total number of cells (STO feeders plus germ cell clumps) was counted and subtracted from the number of mitotically inactive STO feeders initially seeded (i.e. $1.5 \times 10^{5}$ cells). The Institutional Animal Care and Use Committee of the University of Pennsylvania approved all animal protocols (Protocol \#702099).

\subsection{Immunohistochemistry and Immunocytochemistry.} Testes from adult (6-month old) mice were isolated and fixed in $4 \%$ paraformaldehyde. The testes were processed by the Histology and Gene Expression Core at University of Pennsylvania. Tissue sections were deparaffinized, hydrated, and subject to sodium citrate antigen retrieval. Tissue sections were blocked using 10\% normal goat serum followed by $1 \mathrm{hr}$ incubation at room temperature with anti-CXCR4 antibody (BD Bioscience; CD184, clone 2ab11) or a goat immunoglobulin (IgG) antibody. Samples were washed in PBS and incubated for $20 \mathrm{~min}$ with biotin-labeled secondary antibody at room temperature. Sections were washed and incubated with streptavidin-conjugated HRP and then developed using the HistoStain SP substrate kit (Life Technologies).
To examine protein expression in SSC-enriched germ cell cultures, cells were fixed with $4 \%$ paraformaldehyde for $20 \mathrm{~min}$ and permeabilized with $0.1 \%$ Triton X-100 in Dulbecco's PBS for $60 \mathrm{~min}$ at room temperature. After incubating with $20 \%$ normal donkey or goat serum for $60 \mathrm{~min}$ to avoid nonspecific interaction with antibodies, the cells were labeled overnight at $4^{\circ} \mathrm{C}$ with anti-CXCR4 antibody. The following day, cells were incubated with Alexa Fluor 488-conjugated donkey anti-rat IgG secondary antibodies for $5 \mathrm{hr}$ at $4^{\circ} \mathrm{C}$. Nuclei were labeled with $4^{\prime}, 6$-diamidino-2phenylindole (DAPI). The stained cells were analyzed using a Leitz Dialux 20 microscope (Leica Microsystems), and images were obtained with a Spot Insight 2MP Firewire Color Mosaic Digital Camera (Diagnostic Instruments).

2.3. Gene Expression Analysis. Total RNA (1 $\mu \mathrm{g})$ from transfected germ cell cultures or testis was isolated using Trizol and processed for first-strand cDNA synthesis, using TaqMan ${ }^{\circledR}$ Assay Kit (Life Technologies) according to manufacturer directions. Gene expression was assessed by qRT-PCR using commercially available TaqMan Gene Expression primers for mouse GATA4, GDNF, CSF-1, CXCL12, and GAPDH. Gene amplification was quantified using the ABI7500 detection system and accompanying software (Life Technologies). Relative gene expression was quantified using the $2^{\Delta \Delta \mathrm{Ct}}$ method. All data are representative of at least three biological replicates with statistical significance between samples calculated using one-way ANOVA $(p<0.05)$.

2.4. Germ Cell Proliferation and Viability. To test whether CXCR4/CXCL12 regulates in SSC expansion, equal concentration of SSC germ cell culture was plated on STO feeder and treated with the specific CXCR4 inhibitor, AMD3100 $(1.25 \mu \mathrm{M}$; Sigma), or recombinant CXCL12 (10 ng/mL; R\&D Systems), or both. Germ cells were cultured for 7 days after which they were gently dissociated from STO feeders and counted using a hemocytometer. In a repeat experiment, cell viability was assessed using the ATPlite assay kit per manufacturer (Perkin Elmer). In brief, $1 \times 10^{4}$ germ cells were plated onto 96-well plates in defined culture media and treated with AMD3100, or the AKT inhibitor, LY29004 $(33 \mu \mathrm{M})$. At day 4 and day 6 after treatment, cell viability was assessed by quantifying ATP-dependent luciferase activity.

2.5. shRNA Lentiviral Transduction. Lentiviral-mediated short hairpin RNA (shRNA) was used in the stable repression of CXCR4 expression in SSC-enriched germ cell cultures. To create lentiviral particles, commercially obtained pLKO.1puro vectors, containing Cxcr4 shRNA or scrambled control shRNA (Sigma-Aldrich, USA), were cotransfected with envelope (pMD2.G) and packaging (psPAX2) plasmids into HEK293 packaging cells (ATCC) using Lipofectamine 2000 (Life Technologies). A single cell suspension of cultured germ cells was incubated with Cxcr4 shRNA virus or control virus at a multiplicity of infection (MOI) of 5 in the presence of $8 \mu \mathrm{g} / \mathrm{mL}$ polybrene for 18 hours. Cells were allowed to recover for 24 hours following which puromycin $(1 \mu \mathrm{g} / \mathrm{mL})$ 
was used for 72 hours to select cells that stably incorporated the vector.

2.6. Germ Cell Transplantation. As previously described, SSC-enriched germ cell cultures $\left(1 \times 10^{4}\right.$ cells/testis $)$ were transplanted into the testis of adult, Busulfan-treated $(60 \mathrm{mg} / \mathrm{kg})$ 129/SvCP $\times$ C57BL/6 recipient mice (stock no. 101043; The Jackson Laboratory) [18]. A total of 3 recipient mice (i.e., $n=6$ testes) were used per treatment group. The SSC-enriched germ cell cultures derived from ROSA26 mice were used to enable detection of $\beta$-galactosidase activity in recipient testis. Two months after transplantation the testes were harvested and analyzed for donor cell-derived colonies of spermatogenesis by staining with 5-bromo-4chloro-3-indolyl-beta-D-galacto-pyranoside (X-gal; Thermo Fisher Scientific). All X-gal stained colonies that formed within tubules from recipient testis were counted. SSC colony formation was calculated according to the established formula: SSC number per $10^{5} \mathrm{THY}^{+}$cells cultured = (number of donor-derived colonies of spermatogenesis) $\times\left(10^{5}\right.$ total cells harvested $/ 10^{5}$ cells transplanted $) \times\left(1 / 10^{5}\right.$ $\mathrm{THY}^{+}$cells originally cultured).

In vivo investigation of CXCR4-CXCL12 signaling in SSC transplantation assays required repeated injection of AMD3100 into mice. This is due to the short half-life of AMD3100, which is rapidly metabolized and cleared out of the body [19], thus providing an opportunity for donor SSCs to migrate to their stem cell niche using CXCL12/CXCR4 signaling.

2.7. Germ Cell Migration Assay. Culture mouse germ cells were harvested and depleted of feeder cells by two rounds of preplating. $10^{5}$ mouse germ cells in single cell suspension were then placed in the upper chamber of a transwell insert containing a polycarbonate membrane with $5 \mu \mathrm{m}$ pores (Cell Biolabs Inc.). The transwell insert was placed into a well of a 12-well plate containing serum-free medium with or without recombinant CXCL12/SDF-1 (R\&D systems; $10 \mathrm{ng} / \mathrm{mL}$ ) and AMD3100 (Sigma Aldrich, $1.25 \mu \mathrm{M}$ ) [19]. Cultures were incubated for $24 \mathrm{hrs}$ following which the media containing migrated germ cells were collected and cells stained with cell stain solution (Cell Biolabs Inc.). The absorbance of invading germ cells was quantified at OD at $562 \mathrm{~nm}$. Cell stain solution in blank wells containing only medium was used as the reference solution. The higher the number of invading germ cells in the bottom well is, the greater the absorbance quantified is.

\section{Results}

3.1. Expression of CXCR4 and CXCL12 in the Rodent Testes. Prior reports show that the transcript levels of CXCR4 are markedly elevated in mouse $\mathrm{THY}^{+}$enriched SSCs compared to somatic testis cells [10]. SSC-enriched germ cell cultures, but not STO feeder cells, showed strong expression of CXCR4 (Figure 1(a)). Conversely, the CXCL12 ligand was intensely expressed by the STO cell feeder layer (Figure 1(b)). Immunohistochemical (IHC) evaluation of mouse testis showed that
CXCR4 expression was localized to spermatogonia residing on the basement membrane of the seminiferous tubules (Figure 1(c)). Next the gene expression of the Sertoli cell marker, GATA4, was evaluated in relation to GDNF and known GDNF-regulated genes, CSF-1 and CXCL12. Compared to testes from untreated mice, an increase in the expression of GATA4 was correlated with elevations in the expression of GDNF, CSF-1, and CXCL12 in the testis of Busulfan-treated mice (Figure 1(d)).

\subsection{Impact of CXCR4-CXCL12 Signaling on SSC Homeostasis.}

To determine the influence of attenuating CXCR4-CXCL12 signaling on SSC containing germ cell cultures, the cultures were treated with the small molecule inhibitor, AMD3100 [12]. Treatment with AMD3100 had little to no effect on the proliferation or viability of $\mathrm{THY}^{+}$germ cell cultures (Figures 2(a) and 2(b)), whereas treatment with the PI3K-Akt inhibitor, LY29004, dramatically reduced the viability of SSCenriched germ cell cultures (Figure 2(b)).

To further investigate CXCR4-CXCL12 signaling, CXCR4 expression was stably silenced in SSC-enriched germ cell cultures using commercially obtained shRNA lentiviral vectors. Initial screenings evaluated three CXCR4 shRNAs (shCXCR4-1, shCXCR4-2, and shCXCR4-3) and found that shCXCR4-1 and shCXCR4-2 provided the highest efficiency of gene targeting, reducing CXCR4 expression by $\sim 80 \%$ compared to untreated germ cells or germ cells transduced with scrambled control shRNA (Figure 3(a)). Transplantation of the SSC-enriched germ cell cultures into the testes of recipient mice revealed that shRNA-mediated silencing of CXCR4 significantly reduced the number of spermatogenic colonies compared to control donor cells expressing scrambled shRNA ( hCXCR4-1 = 34.3 \pm 11.1 and CXCR4-2 = 32.9 \pm 9.1 versus control $=131.4 \pm 25.9$; mean \pm SEM; $p=0.02$, $n=3$ ) (Figure 3(b)). Next we investigated the importance of CXCR4-CXCL12 signaling in establishing spermatogenesis. Compared to control germ cell culture transplants, germ cell cultures transplanted into recipient testis along with AMD3100 showed a significant reduction in donor-derived spermatogenic colonies $(156.0 \pm 36.4$ versus $62.1 \pm 15.6$ colonies $/ 10^{5}$ cells injected; mean \pm SEM; Student's $t$-test, $p=0.022, n=4$ ) (Figure 3(c)). In a similar experiment, recipient mice were repeatedly administered AMD3100 for 2 days following transplantation of donor germ cell cultures. Compared to untreated recipient controls, the repeated administration of AMD3100 dramatically reduced the number of donor-derived spermatogenic colonies, though this finding was nonsignificant $(156.4 \pm 36.15$ versus $89.9 \pm 12.0$, colonies $/ 10^{5}$ cells injected; mean \pm SEM; Student's $t$-test, $p=0.058, n=4$ replicates) (Figure $3(\mathrm{~d})$ ). These results are in agreement with previous reports and highlight the importance of CXCR4-CXCL12 signaling axis in facilitating recolonization of the testis $[11,12]$.

3.3. CXCL12 Promotes Germ Cell Migration. Although perturbing CXCR4-CXCL12 signaling had minimal effect on in vitro germ cell proliferation, the inhibition of the CXCR4CXCL12 signaling significantly reduced the ability of donorderived SSCs to reestablish spermatogenesis in recipient 

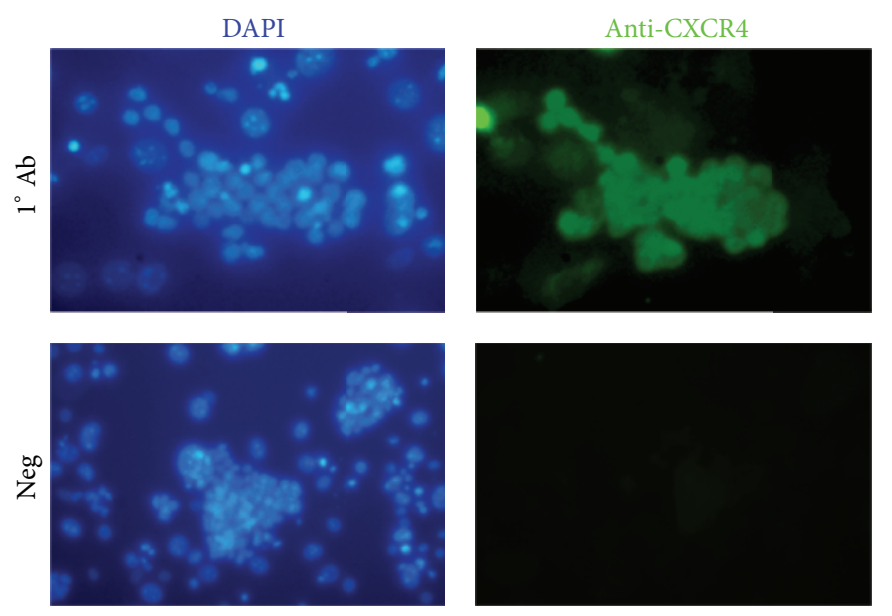

(a)
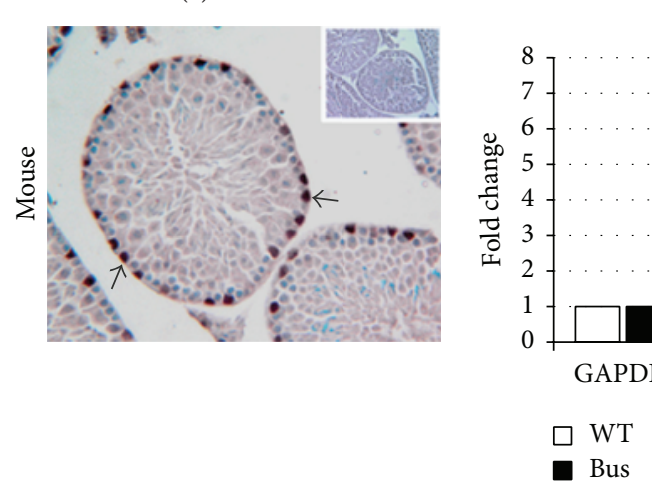

(c)
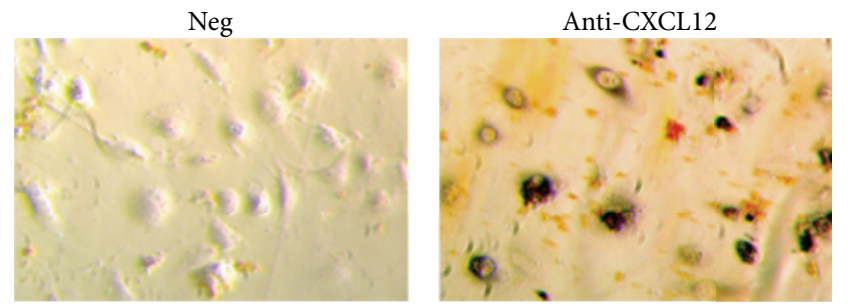

(b)

(d)

FIGURE 1: In vitro and in vivo expression analysis of CXCR4/CXCL12 in rodent testis cells. (a) Expression of CXCR4 membrane receptor in SSC-enriched cultured germ cells was determined by immunofluorescence using FITC-labeled anti-CXCR4 antibody (green), with the cell nucleus labeled by DAPI (blue). (b) Immunohistochemistry staining of CXCL12 protein in the STO feeder cells. (c) Immunohistochemistry staining of CXCR4 in mouse testis. Arrows show CXCR4 staining of germ cells on the basement membrane of seminiferous tubule. (d) The fold change in gene expression of GATA4, GDNF, CSF-1, and CXCL12 in the testes of untreated and Busulfan-treated recipient mice was verified by qRT-PCR. Changes in gene expression are normalized to GAPDH. Significance (denoted as $*$ ) was calculated using Student's $t$-test (with $p<0.05, n=3$ ). Data are means \pm SEM.

testis. Along with recent reports, our findings suggest that CXCR4-CXCL12 signaling has an important role in the homing of SSCs to the stem cell niche [11, 12]. To test this concept in vitro, we established a modified Boydenchamber assay in which germ cell cultures were plated on a polycarbonate membrane insert with a pore size that is $5 \mu \mathrm{m}$ smaller than the diameter of mouse SSCs; therefore, cells located on the bottom well were representative of active cell migration. SSC-enriched germ cell cultures were treated with or without AMD3100, and the transwell insert was incubated in serum-free medium in the presence or absence of CXCL12 $(10 \mathrm{ng} / \mathrm{mL})$. After $24 \mathrm{hrs}$, the number of migrating germ cells was 2.89-fold higher in response to CXCL12 ligand compared to untreated controls (Figure 4). Conversely, inhibition of CXCR4-CXCL12 signaling using AMD3100 lowered the number of migrating germ cells to baseline levels (Figure 4). These results strongly suggest that CXCR4 regulates coordinated motility of undifferentiated germ cells in a CXCL12 ligand-dependent manner.

\section{Discussion}

In this study, we confirm previous findings involving CXCR4CXCL12 signaling and its ability to mediate SSC colonization of the testis $[11,12]$. Compared to controls, reduced colony formation in both CXCR4 shRNA SSCs, as well as those treated with AMD3100, reduced the number of donorderived colonies in recipient testis. Our in vitro migration assay showed that perturbing CXCL12/CXCR4 signaling does not alter SSC proliferation (i.e., self-renewal), but rather this inhibition in signaling reduces SSC ability to migrate. From this finding, we would interpret that the reduced number of donor-derived colonies in AMD3100 treated testis is the result of inhibiting CXCL12/CXCR4 signaling in donor SSCs, which prevents them from relocating to the stem cell niche.

The localization of SSCs within the seminiferous tubules is dynamic and stage-dependent [20, 21]. As such, the processes governing SSCs involve a complex system of soluble signaling factors and cell-cell interactions that enables spatial localization of SSCs and coordinates their ability to self-renew 


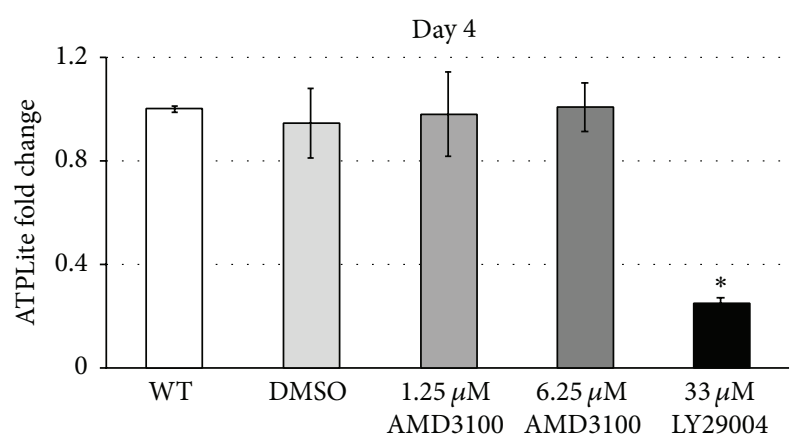

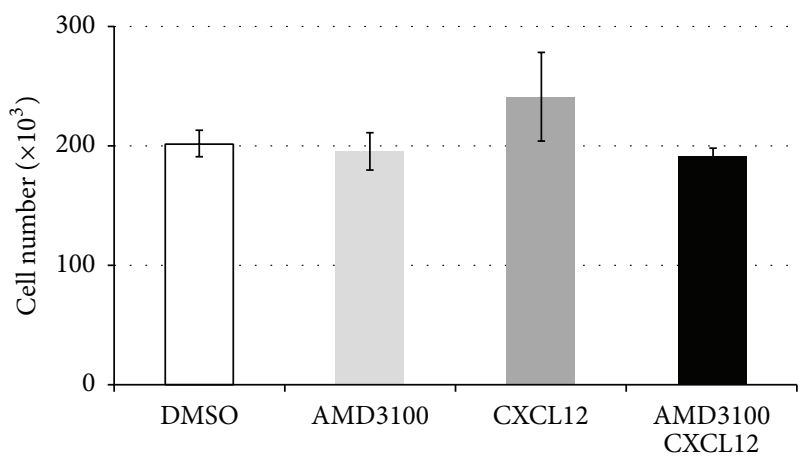

(a)

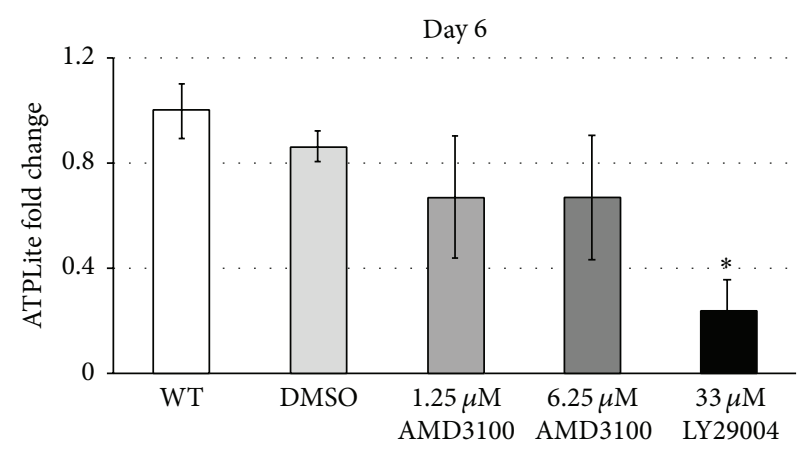

(b)

FIGURE 2: Effects of CXCR4 inhibitor AMD3100 on SSC-enriched THY1 ${ }^{+}$mouse germ cells. (a) An equal number of SSC-enriched cultured germ cells $\left(1 \times 10^{5}\right.$ cells/well) were cultured on a layer of STO feeder cells and were treated with the vehicle (DMSO) or treated with AMD3100 $(1.25 \mu \mathrm{M})$, CXCL12 $(10 \mathrm{ng} / \mathrm{mL})$, or the combination of both CXCL12 and AMD3100. After 7 days the total number of cultured germ cells was quantified by cell counting. Values are representative of three independent experiments, and significance was calculated using one-way ANOVA. Data are means \pm SEM. (b) Impact of AMD3100 on germ cell viability. SSC-enriched cultured germ cells were seeded on feeder-free, laminin-coated 96-well plates at a density of $1 \times 10^{4}$ cells/well. Cells were either untreated (WT) or treated with DMSO control, AMD3100 $(1.25 \mu \mathrm{M}$ and $6.25 \mu \mathrm{M})$, or AKT inhibitor, LY29004 $(33 \mu \mathrm{M})$. Cell viability was assessed at days 4 and 6 following treatment using the ATPLite assay. Data are means \pm SEM, where significance (denoted as $*$ ) was calculated using Student's $t$-test $(p<0.05, n=4)$.

or differentiate [22]. Several cytokines produced by Sertoli cells are strongly implicated in contributing to this signaling, including, CCL9, CXCL5, and CXCL12 [11, 12, 23, 24]. Additionally, the transmigration of SSCs from the lumen of the seminiferous tubule to the SSC niche located on the basement membrane is also regulated by the adhesion molecule $\beta 1$ integrin and small GTP-binding protein RACl (ras-related C3 botulinum toxin substrate 1) [25, 26]. Interestingly, as a member of the Rho family of GTPases, RACl has a role in CXCR4 mediated cellular motility of $\mathrm{CD} 34^{+}$hematopoietic stem cells [27]. Future studies are required to determine whether CXCR4 directed activation of RAC1, or another Rho family of GTPase, functions to mediate SSC transmigration to its respective niche within the basement membrane.

Unlike the Busulfan-treated testes that are devoid of endogenous SSCs, the untreated testes contain both somatic cells and germ cells. Thus, the elevated levels of GATA4, GDNF, CSF-1, and CXCL12 mRNA are likely reflective of the remaining somatic cells that constitute the tubules (e.g., Sertoli cells, peritubular myoid cells). While largely quiescent, SSCs will enter the cell cycle and increase proliferation at a higher frequency following the loss of germ cells following injury [28]. Fittingly, a recent study has shown that cisplatinmediated injury of the mouse testis dramatically increases the production of GDNF [29]. Together with our findings, we would suggest that acute gonadotoxicity in Busulfantreated mouse testes increases production of GDNF, CSF1 , and CXCL12 in response to an absence of germ cells to promote retention of SSCs to its niche on the basement membrane. Because SSCs are enriched for both CSFR1 and CXCR4 expression, it strongly suggests that the donor SSC is readily primed for activation by these ligands $[6,7,12,30$, 31]. As a possible damage response, this mechanism would provide a homing signal for surviving endogenous SSCs to be maintained within the stem cell niche (i.e., SSC homing to the basement membrane mediated by CXCR4-CXCL12 signaling). Subsequently, the elevated production of GDNF and CSF-1, as well as other factors, would promote SSC survival and enhance self-renewal $[6,7,26,30]$. Our finding of elevated transcript levels of GDNF, CSF-1, and CXCL12 in Busulfan-treated testis suggests that this may be the case; however, further studies are required to evaluate protein expression and cellular localization of ligands in somatic cells that constitute the seminiferous tubules. Likewise, additional experiments are needed to track the in vivo spatial of movement of the donor SSC.

In pediatric oncology there is growing concern regarding the gonadotoxic side effects of radiation and chemotherapy 


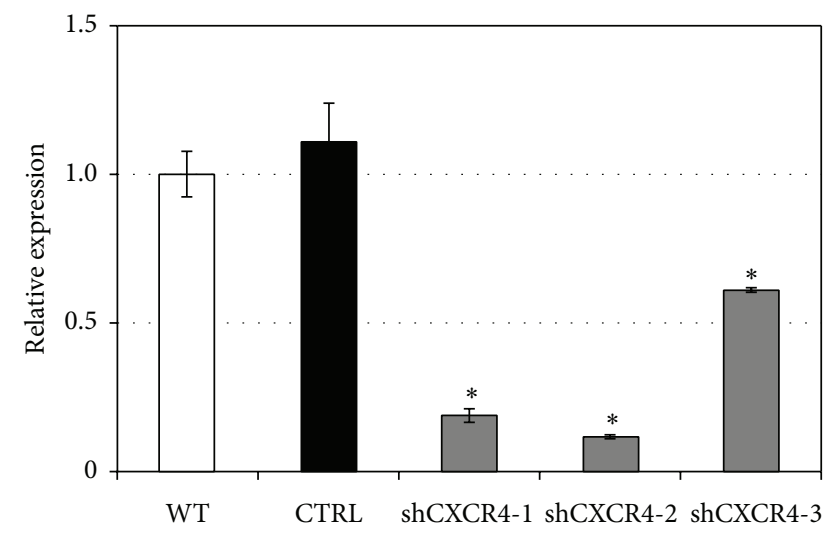

(a)

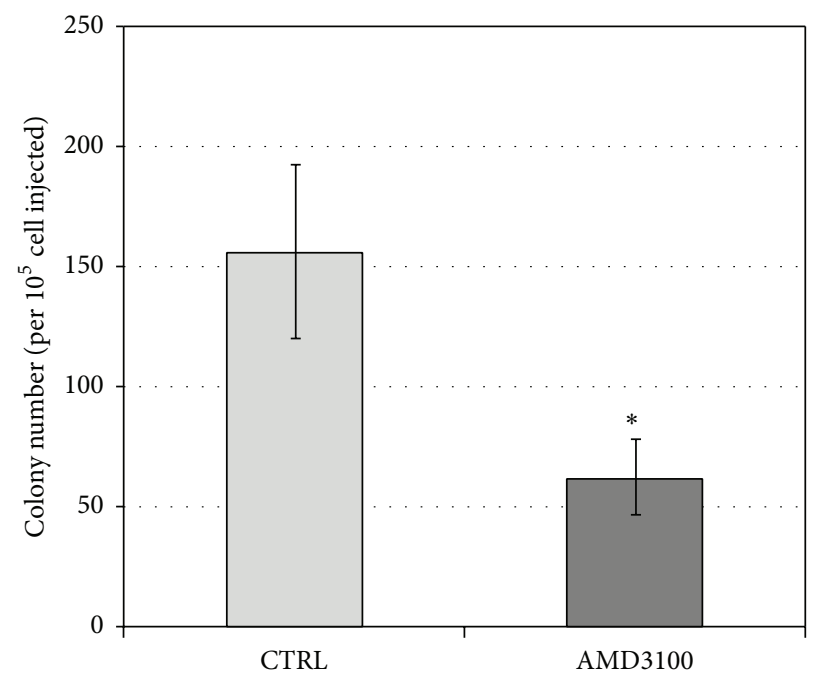

(c)

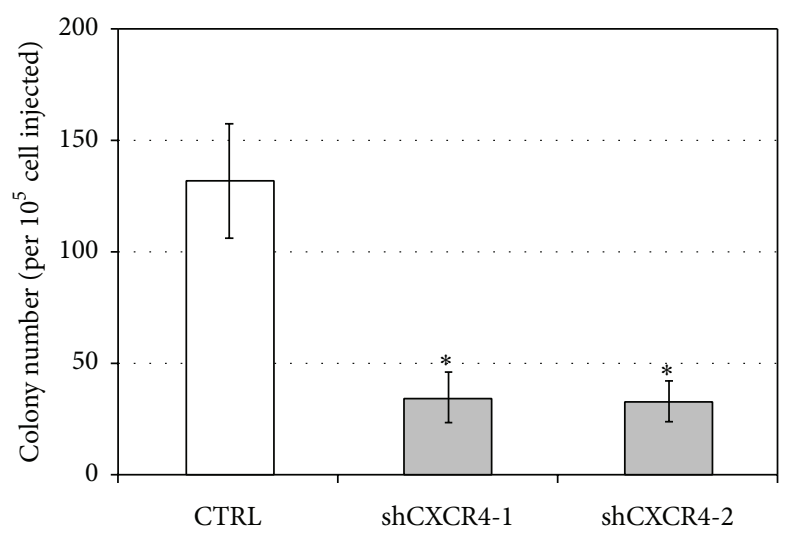

(b)

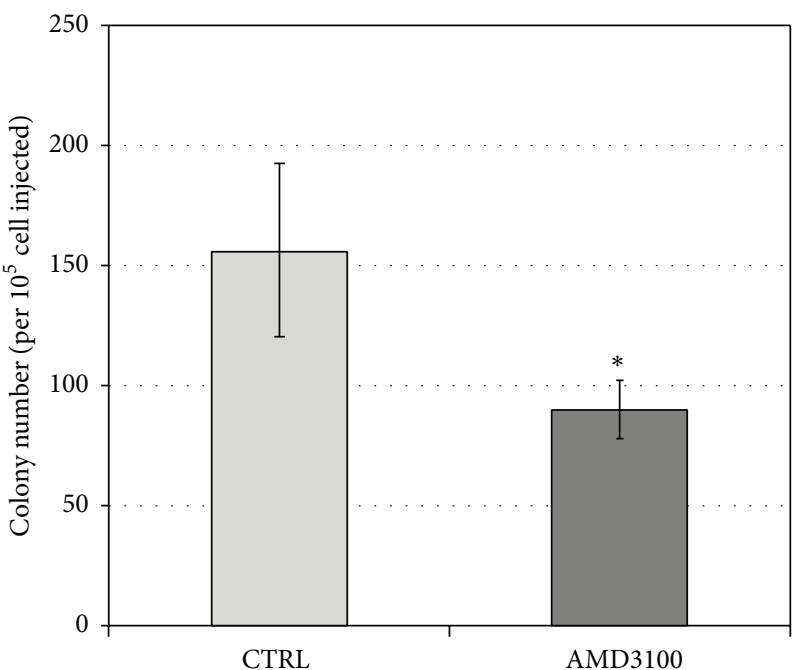

(d)

FIGURE 3: Inhibition of CXCR4 expression or function reduces stem cell activity of SSC-enriched germ cells. (a) qRT-PCR examination of shRNA-mediated knockdown of CXCR4 gene expression. SSC-enriched germ cell cultures were transduced with lentivirus containing a scrambled control shRNA vector or with one of three CXCR4-targeting shRNA clones (shCXCR4-1, shCXCR4-2, and shCXCR4-3). The transcript levels of CXCR4 were normalized to GAPDH gene expression and compared to untreated controls using $2^{-\Delta \Delta C T}$. Significance was calculated using a Student's $t$-test $(p<0.05 ; n=3)$. Data are means \pm SEM. (b) Colony forming efficiency of CXCR4-deficient SSC-enriched germ cell cultures. CXCR4 shRNA lentiviral transduced cultures of SSC-enriched germ cells derived from Rosa-lacZ mice were transplanted at $1 \times 10^{4}$ cells/testis into the testes of Busulfan-treated recipient mice. Donor-derived spermatogenesis was quantified by staining recipient testes with X-gal 2 months after transplantation. Significance was calculated using a Student's $t$-test $(p<0.05 ; n=6$ testes per treatment group). Data are means \pm SEM. (c) AMD3100 disrupts SSC colony formation. Donor Rosa-lacZ SSC-enriched germ cells were treated with AMD3100 ( $5 \mu \mathrm{g} /$ testis) followed by transplantation into recipient testis (data are means \pm SEM, $p=0.022, n=4$ testes per treatment group). (d) Alternatively, recipient mice bearing Rosa-lacZ SSC-enriched transplanted germ cells were repeatedly administered AMD3100 (5 $\mu \mathrm{g} /$ testis) via the intraperitoneal (IP) route at $12 \mathrm{hr}$ intervals for 2 days following transplantation. Donor-derived colonies were detected using X-gal and counted 2 months after transplantation. Data are means \pm SEM, where significance was assessed using Student's $t$-test $(p=0.058, n=2$ testes per treatment group).

on the fertility of prepubertal boys [32-34]. As a result, there are an increasing number of institutions that are offering fertility preservation by cryopreserving testis biopsies for future autologous transplantation of the SSCs [35]. This approach holds great potential as a regenerative stem cell therapy to restore patient fertility but still faces several obstacles, including enrichment of patient SSCs and separation from possible contaminating cancer cells [32, 35]. Barring such obstacles, the possibility of autologous SSC transplantation in patients is close to being realized. In this report, the results of using a Busulfan mouse model suggest another important aspect for clinical consideration: what changes in the expression of essential SSC niche factors occur in response to gonadotoxins or the absence of germ cells? While biochemical evaluation of gonadotropins and androgen levels are essential in evaluating patient recovery from exposure to gonadotoxins, there is a clear lack of knowledge regarding how similar exposures affect the expression of SSC niche factors (e.g., GDNF, CSF-1, 


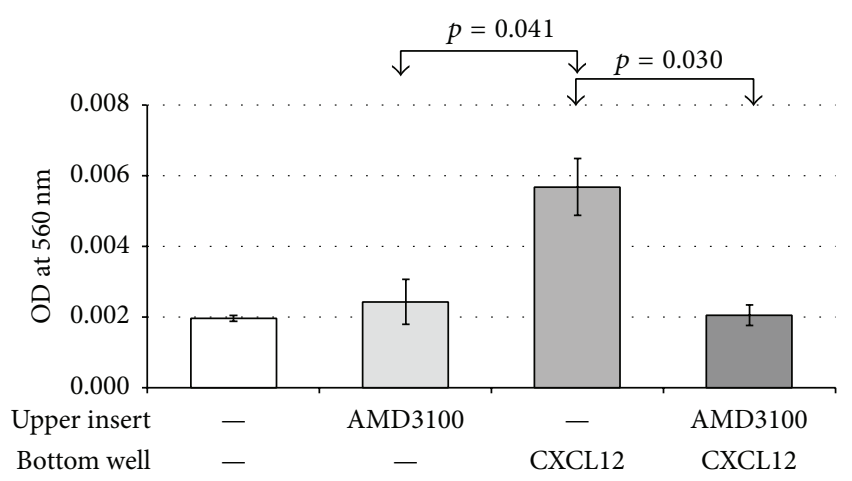

FIGURE 4: CXCL12/CXCR4 pathway promotes germ cell migration. SSC-enriched germ cells seeded in upper transwell in the presence or absence of AMD3100 $(1.25 \mu \mathrm{M})$ were exposed to CXCL12 $(10 \mathrm{ng} / \mathrm{mL})$ in the lower well. Measurement of the absorbance $(562 \mathrm{~nm})$ in the bottom well was used to assess the active number of germ cells that migrated from upper to lower wells. Data are means \pm SEM, where significance was assessed using a Student's $t$-test $(p<0.05, n=3$ experimental replicates).

and CXCL12) [36]. Understanding such changes will improve the clinical success of patients regaining complete fertility following autologous SSC transplantation.

\section{Conclusions}

We show that the CXCR4-CXCL12 signaling axis functions to promote homing of SSCs to the stem cell niche and plays a critical role in reestablishing spermatogenesis. Moreover, we demonstrate that Busulfan treatment of recipient mice increases the basal levels of GDNF, CSF-1, and CXCL12 expression, suggesting that Busulfan-mediated gonadotoxicity alters the testis microenvironment. Additional studies using Busulfan-treated testis or $\mathrm{W} / \mathrm{Wv}$ mouse testis will shed further light on other soluble factors and signaling events that would enable an appropriate response to facilitate recruitment of the SSCs to their niche.

\section{Conflict of Interests}

The authors declare that there is no conflict of interests regarding the publication of this paper.

\section{Acknowledgments}

The authors thank C. Freeman and R. Naroznowski for assistance with animal maintenance. This study was supported by National Institute of Child Health and Human Development Grant HD 052728 (Ralph L. Brinster) and the Robert J. Kleberg, Jr. and Helen C. Kleberg Foundation (Ralph L. Brinster).

\section{References}

[1] R. L. Brinster, "Male germline stem cells: from mice to men," Science, vol. 316, no. 5823, pp. 404-405, 2007.
[2] J. M. Oatley and R. L. Brinster, "The germline stem cell niche unit in mammalian testes," Physiological Reviews, vol. 92, no. 2, pp. 577-595, 2012.

[3] Q.-E. Yang and J. M. Oatley, "Spermatogonial stem cell functions in physiological and pathological conditions," Current Topics in Developmental Biology, vol. 107, pp. 235-267, 2014.

[4] M. D. Griswold and J. M. Oatley, "Concise review: defining characteristics of mammalian spermatogenic stem cells," Stem Cells, vol. 31, no. 1, pp. 8-11, 2013.

[5] E. González-González, P. P. López-Casas, and J. Del Mazo, "Gene silencing by RNAi in mouse Sertoli cells," Reproductive Biology and Endocrinology, vol. 6, article 29, 2008.

[6] H. Kubota, M. R. Avarbock, and R. L. Brinster, "Growth factors essential for self-renewal and expansion of mouse spermatogonial stem cells," Proceedings of the National Academy of Sciences of the United States of America, vol. 101, no. 47, pp. 16489-16494, 2004.

[7] J. M. Oatley, M. R. Avarbock, and R. L. Brinster, "Glial cell linederived neurotrophic factor regulation of genes essential for self-renewal of mouse spermatogonial stem cells is dependent on Src family kinase signaling," Journal of Biological Chemistry, vol. 282, no. 35, pp. 25842-25851, 2007.

[8] X. Meng, M. Lindahl, M. E. Hyvönen et al., "Regulation of cell fate decision of undifferentiated spermatogonia by GDNF," Science, vol. 287, no. 5457, pp. 1489-1493, 2000.

[9] Z. Niu, S. M. Goodyear, S. Rao et al., "MicroRNA-21 regulates the self-renewal of mouse spermatogonial stem cells," Proceedings of the National Academy of Sciences of the United States of America, vol. 108, no. 31, pp. 12740-12745, 2011.

[10] J. M. Oatley, M. R. Avarbock, A. I. Telaranta, D. T. Fearon, and R. L. Brinster, "Identifying genes important for spermatogonial stem cell self-renewal and survival," Proceedings of the National Academy of Sciences of the United States of America, vol. 103, no. 25, pp. 9524-9529, 2006.

[11] M. Kanatsu-Shinohara, K. Inoue, S. Takashima et al., "Reconstitution of mouse spermatogonial stem cell niches in culture," Cell Stem Cell, vol. 11, no. 4, pp. 567-578, 2012.

[12] Q.-E. Yang, D. Kim, A. Kaucher, M. J. Oatley, and J. M. Oatley, "CXCL12-CXCR4 signaling is required for the maintenance of mouse spermatogonial stem cells," Journal of Cell Science, vol. 126, no. 4, pp. 1009-1020, 2013.

[13] B. Westernströer, D. Langenstroth, S. Kliesch et al., "Developmental expression patterns of chemokines CXCL11, CXCL12 and their receptor CXCR7 in testes of common marmoset and human," Cell and Tissue Research, vol. 361, no. 3, pp. 885-898, 2015.

[14] S. Liekens, D. Schols, and S. Hatse, "CXCL12-CXCR4 axis in angiogenesis, metastasis and stem cell mobilization," Current Pharmaceutical Design, vol. 16, no. 35, pp. 3903-3920, 2010.

[15] N. M. Moll and R. M. Ransohoff, "CXCL12 and CXCR4 in bone marrow physiology," Expert Review of Hematology, vol. 3, no. 3, pp. 315-322, 2010.

[16] K. A. Molyneaux, H. Zinszner, P. S. Kunwar et al., "The chemokine SDF1/CXCL12 and its receptor CXCR4 regulate mouse germ cell migration and survival," Development, vol. 130, no. 18, pp. 4279-4286, 2003.

[17] H. Kubota and R. L. Brinster, "Culture of rodent spermatogonial stem cells, male germline stem cells of the postnatal animal," Methods in Cell Biology, vol. 86, pp. 59-84, 2008.

[18] R. L. Brinster and J. W. Zimmermann, "Spermatogenesis following male germ-cell transplantation," Proceedings of the National 
Academy of Sciences of the United States of America, vol. 91, no. 24, pp. 11298-11302, 1994.

[19] J. B. Rubin, A. L. Kung, R. S. Klein et al., "A small-molecule antagonist of CXCR4 inhibits intracranial growth of primary brain tumors," Proceedings of the National Academy of Sciences of the United States of America, vol. 100, no. 23, pp. 13513-13518, 2003.

[20] A. M. Klein, T. Nakagawa, R. Ichikawa, S. Yoshida, and B. D. Simons, "Mouse germ line stem cells undergo rapid and stochastic turnover," Cell Stem Cell, vol. 7, no. 2, pp. 214-224, 2010.

[21] S. Yoshida, M. Sukeno, and Y.-I. Nabeshima, "A vasculatureassociated niche for undifferentiated spermatogonia in the mouse testis," Science, vol. 317, no. 5845, pp. 1722-1726, 2007.

[22] Q.-E. Yang and J. M. Oatley, "Spermatogonial stem cell functions in physiological and pathological conditions," in Current Topics in Developmental Biology, R. Michael, Ed., vol. 107, chapter 9, pp. 235-267, Academic Press, Cambridge, Mass, USA, 2014.

[23] L. Dovere, S. Fera, M. Grasso et al., "The Niche-Derived Glial Cell Line-Derived Neurotrophic Factor (GDNF) induces migration of mouse spermatogonial stem/progenitor cells," PLoS ONE, vol. 8, no. 4, Article ID e59431, 2013.

[24] L. Simon, G. C. Ekman, T. Garcia et al., "ETV5 regulates sertoli cell chemokines involved in mouse stem/progenitor spermatogonia maintenance," STEM CELLS, vol. 28, no. 10, pp. 18821892, 2010.

[25] M. Kanatsu-Shinohara, M. Takehashi, S. Takashima et al., "Homing of mouse spermatogonial stem cells to germline niche depends on $\beta 1$-integrin," Cell Stem Cell, vol. 3, no. 5, pp. 533-542, 2008.

[26] S. Takashima, M. Kanatsu-Shinohara, T. Tanaka, M. Takehashi, H. Morimoto, and T. Shinohara, "Rac mediates mouse spermatogonial stem cell homing to germline niches by regulating transmigration through the blood-testis barrier," Cell Stem Cell, vol. 9, no. 5, pp. 463-475, 2011.

[27] M. Wysoczynski, R. Reca, J. Ratajczak et al., "Incorporation of CXCR4 into membrane lipid rafts primes homing-related responses of hematopoietic stem/progenitor cells to an SDF-1 gradient," Blood, vol. 105, no. 1, pp. 40-48, 2005.

[28] M. L. Meistrich, "Critical components of testicular function and sensitivity to disruption," Biology of Reproduction, vol. 34, no. 1, pp. 17-28, 1986.

[29] J. G. Harman and J. H. Richburg, "Cisplatin-induced alterations in the functional spermatogonial stem cell pool and niche in C57/BL/6J mice following a clinically relevant multi-cycle exposure," Toxicology Letters, vol. 227, no. 2, pp. 99-112, 2014.

[30] J. M. Oatley, M. J. Oatley, M. R. Avarbock, J. W. Tobias, and R. L. Brinster, "Colony stimulating factor 1 is an extrinsic stimulator of mouse spermatogonial stem cell self-renewal," Development, vol. 136, no. 7, pp. 1191-1199, 2009.

[31] X. Wu, S. M. Goodyear, J. W. Tobias, M. R. Avarbock, and R. L. Brinster, "Spermatogonial stem cell self-renewal requires ETV5-mediated downstream activation of brachyury in mice," Biology of Reproduction, vol. 85, no. 6, pp. 1114-1123, 2011.

[32] R. B. Struijk, C. L. Mulder, F. van der Veen, A. M. M. van Pelt, and S. Repping, "Restoring fertility in sterile childhood cancer survivors by autotransplanting spermatogonial stem cells: are we there yet?" BioMed Research International, vol. 2013, Article ID 903142, 12 pages, 2013.

[33] D. Van Saen, H. Tournaye, and E. Goossens, "In search of the most efficient fertility preservation strategy for prepubertal boys," Facts, Views \& Visions in ObGyn, vol. 5, no. 1, pp. 45-58, 2013.

[34] C. Wyns, M. Curaba, B. Vanabelle, A. van Langendonckt, and J. Donnez, "Options for fertility preservation in prepubertal boys," Human Reproduction Update, vol. 16, no. 3, Article ID dmp054, pp. 312-328, 2010.

[35] E. Goossens, D. Van Saen, and H. Tournaye, "Spermatogonial stem cell preservation and transplantation: from research to clinic," Human Reproduction, vol. 28, no. 4, pp. 897-907, 2013.

[36] J. P. Ginsberg, "Gonadotoxicity of cancer therapies in pediatric and reproductive-age males," in Oncofertility Medical Practice: Clinical Issues and Implementation, C. Gracia and T. K. Woodruff, Eds., pp. 15-23, Springer, New York, NY, USA, 2012. 

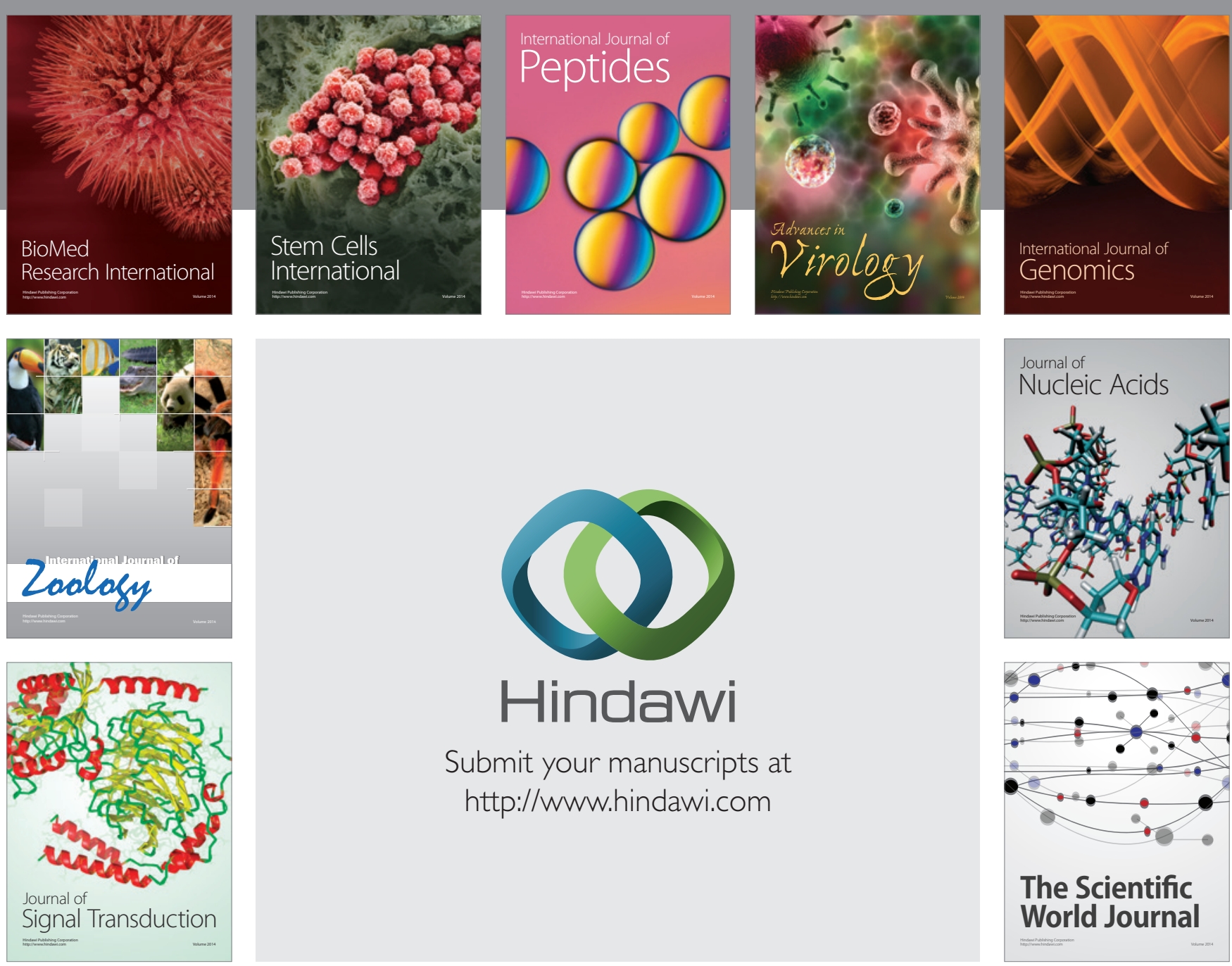

Submit your manuscripts at

http://www.hindawi.com
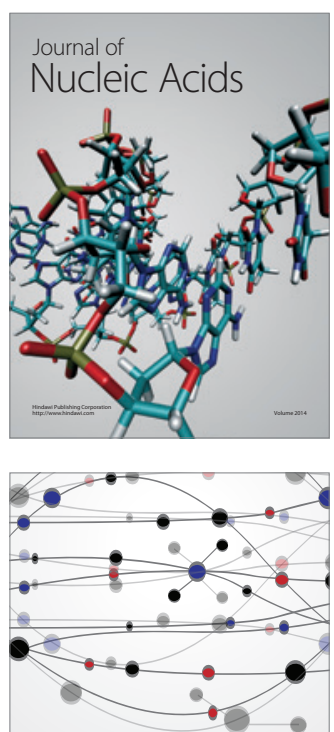

The Scientific World Journal
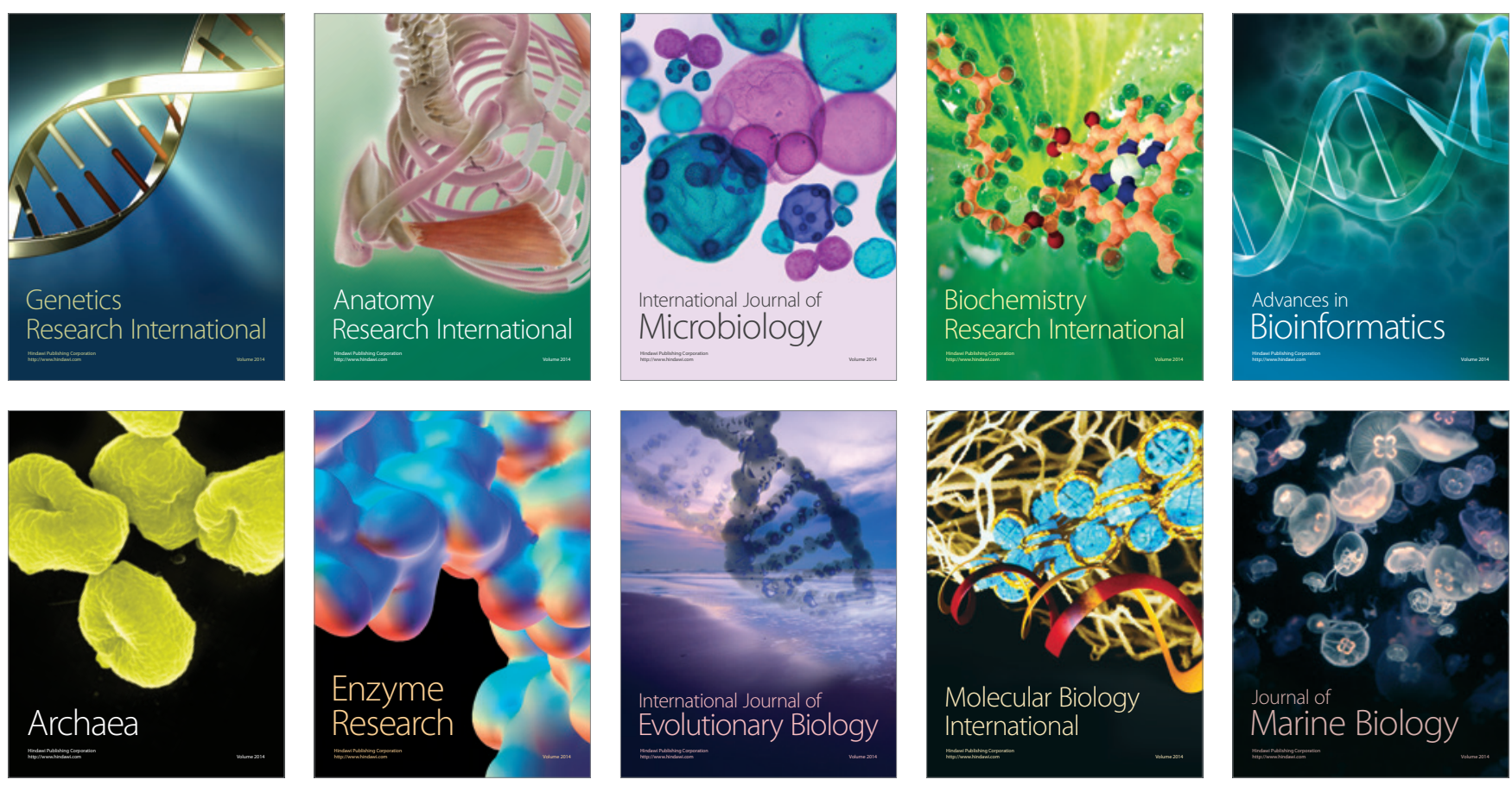